Revista Brasileira de Informática na Educação - RBIE

Brazilian Journal of Computers in Education

(ISSN online: 2317-6121; print: 1414-5685)

http://br-ie.org/pub/index.php/rbie

$\begin{array}{lllr}\text { Submission: } 15 / \mathrm{Feb} / 2017 ; & 1^{\text {st }} \text { round notif.: 27/Oct/2017; } & \text { New version: 10/Dec/2017; } & 2^{\text {nd }} \text { round notif.: 02/May/2018 } \\ \text { Camera ready: 04/Sep/2018; } & \text { Edition review: 13/Sep/2018; } & \text { Available online: 13/Sep/2018; }\end{array}$

\title{
Juiz online no ensino de CS1 - lições aprendidas e proposta de uma ferramenta
}

\author{
Title: Online judge in CS1 education - Lessons learned and proposal of a tool
}

\author{
Rodrigo Elias Francisco \\ Instituto Federal Goiano \\ rodrigo.francisco@ifgoiano.edu.br
}

\author{
Ana Paula Laboissière Ambrósio \\ Universidade Federal de Goiás \\ apaula@inf.ufg.br
}

Cleon Xavier Pereira Júnior

Instituto Federal Goiano

cleon.junior@ifgoiano.edu.br

\author{
Márcia Aparecida Fernandes \\ Universidade Federal de Uberlândia \\ marcia@ufu.br
}

\begin{abstract}
Resumo
Os juízes online, inicialmente usados em maratonas de programação, vêm sendo adotados também para o ensino de Programação Introdutória (CS1), apresentando algumas vantagens, como a redução da carga de trabalho do professor e o feedback instantâneo ao estudante, e desvantagens por não serem totalmente adaptados como ferramenta para o ensino. Este trabalho apresenta uma Revisão Sistemática da Literatura (RSL) acerca de juízes online para o ensino de CS1, que se baseou nos seguintes aspectos: beneficios, problemas; requisitos funcionais e não-funcionais. $O$ resultado da revisão contribuiu para especificar um juiz online com foco no suporte à disciplina de CS1. Também foi realizada uma experiência prática com o uso do juiz online BOCA (desenvolvido para maratonas de programação) no ensino de turmas matriculadas em CS1. As lições aprendidas na experiência prática e os conhecimentos adquiridos na RSL contribuíram para uma proposta de juiz online para o ensino de CS1, com foco principalmente em três requisitos considerados essenciais: construção de listas de exercícios, feedback personalizado, e plágio. Assim, esta pesquisa contribui com o ensino de programação introdutória ao apresentar uma abordagem baseada em resultados encontrados na literatura, através de artigos que apresentam diferentes juízes online para o ensino, e experiências práticas com turmas reais.
\end{abstract}

Palavras-Chave: juiz online; CS1; Programação Introdutória; RSL; Feedback; Plágio.

\begin{abstract}
Online judges, initially used in programming marathons, have also been adopted to teach Introductory Programming (CS1), presenting some advantages, as reducing teacher workload and instant feedback to students, and disadvantages by of not being fully adapted as a tool for teaching. This paper presents a Systematic Review of Literature (SRL) about online judges for teaching CS1, which focused on aspects: benefits, problems; functional requirements and nonfunctional requirements. The result contributed to an online judge specification that meets the CS1 discipline. Also a practical experience was realized with the use of online judge BOCA (developed for programming marathons) in the teaching of CS1's classes. The lessons learned from practical experience and the knowledge gained at SRL contributed to a judge online proposal for teaching CS1, focusing primarily on three requirements considered essential: building exercise lists, personalized feedback, and plagiarism. Thus, this research contributes to the teaching of introductory programming by presenting an approach based on results found in the literature, through articles presenting different online judges for teaching, and practical experiences with real classes.
\end{abstract}

Keywords: Online judge; CS1; Introductory Programming; SLR; Feedback; Plagiarism. 


\section{Introdução}

Formar profissionais para trabalharem com Tecnologia da Informação (TI) é um desafio, é necessário que estes tenham conhecimentos que os permitam se manterem atualizados. Rocha \& França (2016) destacaram que o ensino de habilidades não ligadas diretamente a uma tecnologia faz com que o conhecimento se dissolva mais lentamente, contribuindo para reduzir a obsolescência. A disciplina de programação introdutória, conhecida na literatura como Computer Science 1 (CS1), possibilita trabalhar nesse sentido, pois tem o objetivo de ensinar os alunos a resolverem exercícios com programas de computadores e não é restrita apenas aos aspectos técnicos da programação.

A disciplina de CS1 é geralmente ministrada no primeiro semestre de cursos da área de Computação e traz diversos desafios para os docentes. Há, por exemplo, o problema das altas taxas de reprovação, o que pôde ser evidenciado por Watson \& Li (2014), que, em uma Revisão Sistemática da Literatura (RSL), verificaram uma taxa de 32,3\%, e Bosse \& Gerosa (2015), que encontraram, nos dados de 2010 a 2014 da Universidade de São Paulo (USP), um percentual médio de reprovações e trancamentos de 29,31\%. Parte do problema pode estar ligado ao planejamento e correção de exercícios, pois estes geram uma sobrecarga no professor, o que dificulta o feedback aos alunos e pode causar frustrações no processo de ensino-aprendizagem (Vier, Gluz, \& Jaques, 2015).

Diversos softwares vêm sendo propostos para melhorar o ensino, porém, seus requisitos são naturalmente relacionados a aspectos pedagógicos, trazendo um cenário de pesquisas multidisciplinar. No caso da disciplina de CS1, é importante considerar, por exemplo, que programar exige elevado nível de generalização, abstração e pensamento crítico (Gomes, Areias, Henriques, \& Mendes, 2008), que necessita de certos modelos mentais (Byrne, \& Lyons, 2001), e que sofre influência da motivação (Rountree, Rountree, Robins, \& Hannah, 2004).

É necessário que os alunos de CS1 resolvam uma grande quantidade de exercícios, o que motiva a utilizar corretores automáticos denominados juízes online. Um juiz online oferece exercícios para serem resolvidos com a submissão de códigos-fonte, em uma linguagem de programação, e os corrige automaticamente (Kurnia, Lim, \& Cheang, 2001) usando casos de teste. Cada caso de teste possui um conjunto de entradas e suas respectivas saídas. Para verificar se a resposta de um exercício é uma solução correta, um programa passa por seu respectivo conjunto de testes. Estes corretores automáticos reduzem a carga de trabalho do professor e possibilitam investir mais nas questões pedagógicas.

Um levantamento sobre juiz online no ensino de CS1 e o uso do sistema BOCA (Campos \& Ferreira, 2004) em turmas de CS1 ofereceram uma perspectiva teórica e prática que levaram à proposta preliminar da ferramenta pedagógica apresentada neste artigo. $\mathrm{O}$ artigo está organizado em cinco seções. A Seção 2 aborda Trabalhos Relacionados, descrevendo o tema de maneira abrangente. A Seção 3 apresenta algumas lições aprendidas com a experiência de uso do BOCA em turmas de CS1. Esta experiência permitiu comparar o uso de juiz online para o ensino em relação aos utilizados em competições. A Seção 4 apresenta uma proposta preliminar de um juiz online para o ensino de CS1, atacando aspectos considerados necessários e emergenciais, levantados a partir da RSL e das lições aprendidas. Por fim, a Seção 5 apresenta as considerações finais. 


\section{Trabalhos Relacionados}

Uma RSL realizada em 2016 teve como objetivo identificar "Qual a especificação mais adequada para um juiz online na disciplina de CS1?” (Francisco, Pereira Júnior, \& Ambrósio, 2016). Os trabalhos usados na síntese estão listados, em ordem cronológica, na tabela 1. A revisão foi norteada por quatro questões:

QP1 - Quais os benefícios ao usar juiz online no ensino de CS1?

QP2 - Quais os problemas ao usar juiz online no ensino de CS1?

QP3 - Quais requisitos funcionais um juiz online precisa atender para ser usado no ensino de CS1?

QP4 - Quais requisitos não-funcionais um juiz online precisa atender para ser usado no ensino de CS1?

Tabela 1: Publicações utilizadas na revisão sistemática

\begin{tabular}{|c|c|c|}
\hline ID & Titulo & Referência \\
\hline E1 & $\begin{array}{l}\text { Automatically assessed electronic exams in programming } \\
\text { courses. In Proceedings of the Australasian Computer } \\
\text { Science Week Multiconference (p. 11). ACM. }\end{array}$ & $\begin{array}{l}\text { Rajala , Lindén, } \\
\text { Kurvinen, Laakso \& } \\
\text { Salakoski (2016) }\end{array}$ \\
\hline $\mathrm{E} 2$ & $\begin{array}{l}\text { Are automated assessment tools helpful in programming } \\
\text { courses }\end{array}$ & $\begin{array}{l}\text { Pettit, Homer, } \\
\text { Holcomb, Simone \& } \\
\text { Mengel (2015) }\end{array}$ \\
\hline E3 & $\begin{array}{l}\text { Introduction of the automated assessment of homework } \\
\text { assignments in a university-level programming course. }\end{array}$ & $\begin{array}{l}\text { Poženel, Fürst \& } \\
\text { Mahnič (2015) }\end{array}$ \\
\hline $\mathrm{E} 4$ & Teaching novice programmers using ProgTest. & $\begin{array}{l}\text { Souza, Isotani \& } \\
\text { Barbosa }\end{array}$ \\
\hline E5 & $\begin{array}{l}\text { An Empirical Study of Iterative Improvement in } \\
\text { Programming Assignments. }\end{array}$ & $\begin{array}{l}\text { Pettit, Homer, Gee, } \\
\text { Mengel \& Starbuck } \\
(2015)\end{array}$ \\
\hline E6 & $\begin{array}{l}\text { YOJ: An online judge system designed for programming } \\
\text { courses. }\end{array}$ & Sun \& Jiao (2014) \\
\hline E7 & $\begin{array}{l}\text { Test my code: An automatic assessment service for the } \\
\text { extreme apprenticeship method. }\end{array}$ & $\begin{array}{l}\text { Vihavainen, } \\
\text { Luukkainen \& Pärtel } \\
\text { (2013) }\end{array}$ \\
\hline E8 & FLOP, a free laboratory of programming. & $\begin{array}{l}\text { Llana, Martin- } \\
\text { Martin \& Pareja- } \\
\text { Flores (2012) }\end{array}$ \\
\hline E9 & Jutge.org: an educational programming judge. & $\begin{array}{l}\text { Petit, Giménez \& } \\
\text { Roura (2012) }\end{array}$ \\
\hline E10 & $\begin{array}{l}\text { Ability-training-oriented automated assessment in } \\
\text { introductory programming course. }\end{array}$ & $\begin{array}{l}\text { Wang, Su, Ma, } \\
\text { Wang \& Wang } \\
(2011)\end{array}$ \\
\hline E11 & $\begin{array}{l}\text { An automated assessment and reporting tool for introductory } \\
\text { Java programs. }\end{array}$ & $\begin{array}{l}\text { AlShamsi \& Elnagar } \\
\text { (2011) }\end{array}$ \\
\hline E12 & $\begin{array}{l}\text { Incorporating an Automatic Judge into Blended Learning } \\
\text { Programming Activities. }\end{array}$ & $\begin{array}{l}\text { Georgouli \& } \\
\text { Guerreiro (2010) }\end{array}$ \\
\hline
\end{tabular}

\subsection{Benefícios ao usar juiz online no ensino de CS1}

Um dos principais benefícios de um sistema de correção automática é a facilidade de elaboração e correção de listas de exercícios de programação. Isso diminui a carga de trabalho do professor (E1, E10, E11). A maioria dos sistemas tem um banco de questões associado, que permite a fácil elaboração de extensas listas de exercícios (E9). Visto que este banco de questões é desenvolvido com objetivos pedagógicos e com alto potencial de reuso, os exercícios tendem a ser melhor preparados, trazendo benefícios ao ensino (E6, E8). Muitos exercícios vêm de livros usados em 
cursos introdutórios de programação (E4). Em diversos casos, os exercícios são classificados de acordo com os conceitos envolvidos e o grau de dificuldade. No caso dos exercícios criados no sistema FLOP, a classificação levou em consideração os níveis básicos de domínio cognitivo da taxonomia de Bloom (E8). Essa classificação permite adaptar a escolha dos exercícios ao nível de conhecimento do aluno.

Do ponto de vista pedagógico, os sistemas de correção automática podem ser catalisadores de mudanças na forma de ensinar e aprender programação. A facilidade de obter feedback no momento que o aluno precisa permite que ele prossiga no seu ritmo (E8), e promove a autoaprendizagem (E10) ao trazer aspectos da sintaxe, estrutura, semântica e resultados de testes no feedback dado. Isso pode ter um impacto na motivação dos alunos (E12). Professores também podem mudar o formato das aulas para incorporar o uso de juízes online (E1, E7). Estas ferramentas também podem ser usadas para automatizar a execução e correção de exames. $\mathrm{O}$ ambiente controlado necessário para o uso da ferramenta ViLLE em exames contribui para uma baixa ocorrência de plágio (E1).

Por possuir um banco de dados de soluções, o uso de juízes online colabora com o monitoramento do progresso do aluno (E11), possibilitando aos docentes uma visão global e temporal do desenvolvimento dos alunos na disciplina. $\mathrm{O}$ fato do sistema armazenar as soluções submetidas para os exercícios propostos permite a análise com objetivos de pesquisa e de adaptações no ensino.

A assiduidade e a transparência também são benefícios educacionais apontados (E12). A assiduidade é justificada pela facilidade do sistema controlar os prazos e a transparência se refere ao fato de que não há interferência de fatores subjetivos na correção dos exercícios.

\subsection{Problemas ao usar juiz online no ensino de CS1}

Grande parte dos problemas identificados ao usar sistemas de correção automática no ensino de CS1 estão relacionados à falta de maturidade destas ferramentas e à dificuldade de instalação e manipulação dos programas (E3, E11). A falta de validação dos sistemas é um problema apontado em várias pesquisas (E2, E3, E5, E8). Existe também a preocupação com segurança e escalabilidade (E9), que impacta na gestão da infraestrutura. Além disso, existem problemas de usabilidade (E4), pois houve estudantes que apresentaram dificuldades no processo de submissão nas primeiras tentativas e que consideraram o feedback da ferramenta insuficiente.

Outra fonte importante de problemas é a percepção dos alunos, e como estes interagem com os sistemas. Por exemplo, pode haver problemas entre os alunos quando as notas deles são visíveis para todos (E12). Também, alunos podem não gostar de sistemas contextualizados em competições. O vocabulário de concursos e ranking torna os alunos mais competitivos (E12), o que pode ser encarado de maneira negativa. Por outro lado, há a ideia de que as competições no ensino de CS1 são úteis para que alunos melhorem as habilidades de programação (E6). Opiniões divergentes indicam que o problema deve ser investigado mais a fundo, pois está ocorrendo com frequência o uso da gamificação em tecnologias educacionais.

A questão de como ou quanto os sistemas de juiz online contribuem na melhoria dos resultados dos alunos ainda está em aberto. Do ponto de vista dos alunos, ainda não existe uma visão clara sobre a contribuição do uso destes sistemas no seu aprendizado (E2, E12). Além disso, não parece haver aumento das notas dos alunos, com o uso destes sistemas (E10).

Outro problema importante é o plágio. Apesar deste não ser um problema exclusivo dos sistemas de juiz online, o fato das atividades muitas vezes serem feitas em casa (E3), seu peso na nota e a grande quantidade de exercícios influenciam na ocorrência de plágio. No entanto, o fato da verificação ser automática permite a inclusão de verificadores de plágio nos sistemas. 
Por fim, uso de ambientes de desenvolvimento e o fato do sistema fazer a correção automática pode levar a uma prática de programação baseada em tentativa e erro. Os alunos acabam confiando nos testes apresentados pela ferramenta e não fazem seus próprios testes (E7).

\subsection{Requisitos Funcionais para um juiz online ser usado no ensino de CS1}

A leitura dos trabalhos sobre $\mathrm{CS} 1$ e juiz online trouxe quatro conjuntos de requisitos funcionais: feedback (E1, E3, E4, E5, E6, E7, E8, E9, E10, E11, E12), Integração do Sistema com os Cursos (E1, E3, E4, E6, E7, E8, E9, E10), Análise do Desempenho Geral dos Alunos (E7, E9, E11), e Oferecer Diferentes Tipos de Atividades (E1, E3, E4, E6, E7). A tabela 3 (seção 4) apresenta, de forma resumida, as ferramentas e os requisitos funcionais que implementam. As referências permitem que interessados em conhecer detalhes específicos das ferramentas possam fazê-lo. Neste trabalho, optou-se por uma visão transversal das mesmas, permitindo uma análise comparativa de como estes requisitos funcionais foram abordados e que são descritos a seguir.

Os requisitos necessários para que o sistema atenda à disciplina de CS1 dependem de cada contexto de ensino. Respondendo à QP3, o ideal é um sistema que atenda aos requisitos identificados, mas que permita ao professor configurar quais recursos deseja usar.

O feedback é fundamental para a aprendizagem do aluno e é o requisito chave dos sistemas de correção automática de algoritmos. Normalmente, o feedback é dado a partir dos resultados de testes feitos pelo sistema com o programa submetido pelo aluno (E1, E3, E6, E8, E9, E10). Entretanto, foram criadas estratégias para gerar feedback com pontuação parcial a partir de resultados dos casos de testes (E5, E7). Também foram propostas as possibilidades de professores darem feedback (E12, E6, E7) e de outros alunos darem feedback (E6). Além disso, um sistema que esconde o feedback imediato devido ao uso em exames foi projetado (E1).

As descrições dos erros de compilação, execução e da saída do programa foram usadas para compor o feedback (E1). Foi proposto, neste caso, um feedback que apresenta detalhamento dos casos de teste que produziram erro ao executar o programa submetido pelo (E1). Já a ferramenta ProgTest é baseada em conceitos de teste. Neste caso, foi criada uma estratégia de avaliação em que o programa do aluno é analisado por casos de teste. A primeira etapa avalia se o programa está correto comparado aos casos de testes criado pelos próprios estudantes. Posteriormente também é avaliado se há erro no programa comparando aos casos de testes criados pelo professor (E4).

O juiz online AutoLEP propõe um feedback que considera aspectos de sintaxe e, com isto, é capaz de identificar pequenos casos de erros de sintaxe e lógicos (E10). Houve também a produção de feedback que consideram aspectos estruturais que se relacionam com a semântica (E10, E11). O AutoLEP consegue avaliar situações em que o aluno engana o sistema, como quando fazem um extenso programa que imprime diretamente os resultados sem usar estruturas indicadas, por meio de comparação de um grafo que representa o programa com modelos armazenados ligados à especificação do exercício. O sistema eGrader verifica se o código-fonte corresponde à resposta correta por meio de semelhança estrutural considerando o grafo que representa o programa e métricas de software. Já no sistema Athene (E5), buscaram mostrar no feedback a evolução do estilo de codificação usado pelo aluno no tempo, permitindo identificar os casos em que o aluno acerta $100 \%$ da questão com um programa escrito com código muito complexo.

Alguns trabalhos se preocuparam em integrar o sistema de juiz online com outras ferramentas usadas nos cursos. O Test MyCode foi projetado para uso em Massive Open Online Courses (MOOC's) com um viés pedagógico de aprendizagem extrema (E7). Trata-se de um sistema, na forma de plugin para um IDE, que visou não sobrecarregar os alunos, se relacionar com a realidade de desenvolvimento de software de indústria, oferecer pontos parciais para exercícios, ser de fácil gestão por professores e aprimorar práticas de engenharia de software dos 
alunos. Já o projeto do juiz online Jutge.org (E9) considerou ideias de sistemas de gestão de aprendizagem, que possibilitam aos professores gerenciar cursos, adicionar exercícios, anexar documentos, criar listas de exercícios, monitorar o progresso dos alunos, interagir com alunos, gerenciar lista de alunos e tutores, criar trabalhos, criar concursos, criar exames e orientar alunos quanto às dificuldades nos exercícios. A funcionalidade de gerir cursos foi tratada nos trabalhos (E4, E6). O sistema YOJ (E6) possibilita ao professor configurar a página inicial do curso, gerenciar o material didático do curso, adicionar alunos ao curso, adicionar exercícios para serem resolvidos em sala de aula e em casa, definir atributos relacionados aos exercícios como dados para testá-los e definir a abordagem de teste. A possibilidade de professores cadastrarem novos exercícios foi verificada nos trabalhos (E8, E4).

Outras características com maior impacto aos alunos foram encontradas nos trabalhos. $\mathrm{O}$ uso dos sistemas em exames é apresentado nos trabalhos (E1, E9, E10). A detecção de plágio foi executada nos trabalhos (E3, E6). Houve o uso do programa MOSS (E3) para detecção de plágio. A possibilidade de alunos entrarem no sistema sem cadastro foi verificada (E8, E9). A ferramenta ProgTest (E4) oferece a nota do aluno na solução de um exercício e, para isso, realiza cálculos a partir de casos de teste do aluno contra o programa do aluno, casos de teste do instrutor contra o programa do aluno, e casos de teste do aluno contra o programa do instrutor. Houve o uso do sistema por alunos para resolverem atividades para casa (E3, E6). Um fórum (E6) foi criado para discutir os exercícios, o que proporcionou aos professores premiarem os alunos dispostos a ajudar os colegas respondendo às perguntas.

A classificação de exercício por nível de dificuldade foi realizada (E1, E9). O cálculo do nível de dificuldade dos exercícios foi proposto usando pontuações individuais, contagens de submissão e tempo gasto em cada atividade (E1). Houve a organização dos exercícios por tópicos (E9).

Os trabalhos analisaram o desempenho geral dos alunos. A geração de relatórios com o desempenho de alunos específicos foi proposta (E7, E9, E11). Entretanto, houve a geração de relatórios com o desempenho de turmas (E11).

Exercícios que exigem dos alunos sua resolução por meio da escrita de programas são os mais comuns nos juízes online. Porém, alguns tipos de atividades, diferentes das normalmente encontradas nos corretores automáticos, foram identificadas.

Exercícios que possuem parte do código fonte escrito e solicitam ao aluno que resolva o restante foram criados (E1, E6). Isso permite avaliar do aluno conhecimentos específicos por obrigá-lo a seguir o desenvolvimento da forma que foi iniciado (E6), facilitando a avaliação do entendimento de conceitos abstratos (e.g. matrizes, arquivos e recursividade).

Os sistemas foram usados no contexto de Orientação a Objetos (E3), solicitando que alunos criassem classes com métodos que seguissem assinaturas pré-definidas para que pudessem ser testados. Por outro lado, exercícios que exigiam dos alunos o envio dos casos de teste para os programas feitos foram propostos (E1) e, na intenção de introduzir o conceito de Desenvolvimento Orientado por Testes, foi apresentado para o aluno um conjunto de testes para serem usados localmente na solução dos exercícios (E7).

Foram encontrados exercícios de CS1 que não seguem a estrutura convencional, como os que se relacionam com computação gráfica (E3) e os que são dispostos nos formatos quiz, code shuffling, exercício robô e ligue os itens (E1). Exercícios do tipo quiz possuem questões de múltipla escolha e perguntas abertas curtas. Atividades do tipo Exercício Robô solicitavam que os alunos escrevessem um programa Java que controla um guindaste para mover caixas para uma posição solicitada. Problemas do tipo Code shuffling solicitavam que os alunos ordenassem as linhas de código embaralhadas que resolvem determinado problema. Atividades do tipo Ligue os 
Itens pediam aos alunos para arrastarem os itens na coluna direita aos locais corretos na coluna esquerda.

\subsection{Requisitos não-funcionais para o juiz online ser usado no ensino de CS1}

A necessidade de integração entre sistemas de juiz online com outros sistemas é tratada nos trabalhos (E7, E12). O sistema test mycode (E7), por exemplo, foi desenvolvido como um plugin para ser integrado a um IDE padrão de indústria. Há também a integração do sistema de juiz online mooshak com plataforma de e-learning Caroline (E12).

A usabilidade é outro requisito não-funcional importante no projeto de sistemas para a educação, e foi levada em consideração no sistema FLOP (E8). O sistema foi construído para ser multi-idiomas e afirma ter uma interface simples, com três menus somente na tela principal, o que facilita o processo de memorização por parte do usuário.

Existe um grande interesse em desenvolver sistemas de correção automática de algoritmos para serem amplamente usados na Internet. Alguns trabalhos abordam a questão da segurança (E1, E3, E8, E9). No sistema FLOP (E8), a avaliação das soluções enviadas pelos usuários é feita em um ambiente seguro e controlado para tentar impedir que programas mal-intencionados possam danificar o servidor. Existem limites no tempo de uso da Unidade de Processamento Central (CPU) e no uso de memória pelos programas para evitar bloqueios do sistema, de forma que programas que excedam os limites estabelecidos são mortos. O tempo de execução de processos é controlado. Programas que não fazem nenhuma computação útil são mortos. Além disso, existe um controle no acesso pelos programas dos alunos ao sistema de arquivos e aos recursos de rede. Para lidar com a segurança, os programas submetidos pelos alunos são executados em um servidor Linux através de uma conta de usuário sem privilégios (E3). O projeto ViLLE (E1), usado em exames, lida com a segurança ao solicitar a supervisão da sala de aula, usar firewall para restringir o acesso à Internet e permitir acesso somente ao sistema e à API Java.

Diferente de outros projetos, o sistema Jude.org (E9) executa todos os códigos-fonte enviados, que podem inclusive incluir códigos maliciosos. Para isso, foi necessário limitar o número de submissões permitidas e criar uma infraestrutura com um sistema distribuído em seis servidores Linux. Nesta infraestrutura, o mestre, único que se conecta à internet, executa o servidor web, o Sistema Gerenciador de Banco de Dados e mantém a fila de submissões, enquanto os outros servidores, máquinas escravas criadas com máquinas virtuais, processam as filas de submissões e se conectam ao mestre a partir de uma rede privada.

A escalabilidade foi levada em consideração (E3, E9). A infraestrutura proposta para o sistema Jude.org (E9) faz com que o tempo de resposta do sistema seja quase real, em menos de dez segundos para retornar feedback, tornando o sistema altamente escalável e possibilitando-o a atender a uma crescente quantidade de usuários. Houve a definição de limites de tempo nas execuções dos programas submetidos pelos alunos e de consumo de memória na Máquina Virtual Java (E3).

A disponibilidade foi verificada (E6, E7, E8). Houve o interesse em permitir o acesso por um número muito grande de alunos em qualquer lugar e momento (E8, E6), e também integrado a MOOC's (E7).

\section{Uso do BOCA em turmas de CS1 - Lições Aprendidas}

O BOCA é um sistema de juiz online usado nas maratonas de programação organizadas pela Sociedade Brasileira da Computação (SBC). Um projeto de pesquisa, realizado entre 2015 e 2016, objetivou adaptá-lo ao ensino de CS1, e para isso houve o uso do sistema e modificações em sua implementação, contribuindo para que algumas lições fossem aprendidas. 
Um repositório com 100 exercícios de CS1 foi criado e verificou-se a necessidade de classificá-los quanto aos tópicos abordados e aos níveis de dificuldade a fim de permitir que o sistema fosse capaz de gerar listas de exercícios para os alunos resolverem com base em parâmetros definidos pelo professor.

Estudar como medir a dificuldade de exercícios de CS1 permitiu verificar a complexidade existente na validação. Alunos e professores realizaram uma classificação dos exercícios, o que mostrou que a dificuldade percebida pelos alunos não foi a mesma percebida pelo professor. Uma proposta para automatizar a classificação de dificuldade foi feita, levando em consideração o número de assuntos do exercício e a estrutura do programa que o implementa. No entanto, as turmas são heterogêneas quanto às capacidades dos alunos, o que faz necessário estender este estudo para levar em consideração capacidades individuais, como por exemplo o nível de conhecimento do individuo, ao gerar as listas.

Durante o experimento foi verificado um alto índice de plágio pelos alunos, o que pôde ter sido facilitado pelo uso do BOCA que permitia aos alunos se autenticarem com usuário e senha de colegas e baixarem os códigos-fonte submetidos. Isso motivou investir na pesquisa sobre estratégias para apoiar a identificação de plágio em códigos-fonte de atividades de CS1. Não é comum entre os sistemas de juiz online oferecerem o apoio à identificação de plágio, sendo que para fins pedagógicos pode ser interessante ter estas informações.

Ao proceder às modificações no BOCA, verificou-se a dificuldade de modificar o códigofonte da ferramenta. É um sistema implementado em PHP com o banco de dados PostgreSql e executado sobre Linux e Apache, porém não possui documentação com diagramas que facilitem sua compreensão. Assim, trabalhos futuros devem pesar as vantagens de reutilizar um sistema contra o investimento de desenvolver um novo.

Quanto ao feedback oferecido pelo sistema, ele é restrito a apenas algumas mensagens (como aceito, rejeitado, erro de sintaxe e erro de saída), o que é suficiente para competições. Para fins pedagógicos, as mensagens poderiam ser mais dinâmicas e personalizadas, levando em consideração características dos estudantes.

O uso de Ambiente Virtual de Aprendizagem (AVA) pela Instituição de Ensino mostrou a necessidade de sua integração com o corretor automático. O juiz online precisa estar preparado para essa comunicação, podendo ser implementado via Web Service e considerando escalabilidade e segurança. Verificou-se também que um juiz online didático pode apoiar outras disciplinas, como Estrutura de Dados, Programação Orientada a Objetos e até Computação Gráfica.

A Tabela 2 apresenta uma lista de características identificadas como importantes em um juiz online para o ensino, fazendo um comparativo com os sistemas utilizados em competição. Vale salientar que os resultados apresentados na tabela é uma sumarização das experiências obtidas em turmas iniciantes de programação, através de proposições de exercícios para serem resolvidos e submetidos a um juiz online, junto com informações adquiridas pela Revisão Sistemática da Literatura. 
Tabela 2: Comparação entre juiz online para competição x ensino

\begin{tabular}{|l|l|l|}
\hline \multicolumn{1}{|c|}{ Característica } & \multicolumn{1}{|c|}{ Juiz online para competição } & \multicolumn{1}{|c|}{ Juiz online para o ensino } \\
\hline Geração de listas de exercícios & Desnecessário & Necessário \\
\hline Feedback & Estático & Dinâmico e personalizado \\
\hline Nível de dificuldade & Definido pelos organizadores & Precisa ser calculado pelo sistema \\
\hline Plágio & Desnecessário & Necessário \\
\hline Integração do Sistema com os Cursos & Desnecessário & Necessário \\
\hline Usabilidade & Desnecessário & Necessário \\
\hline Manutenibilidade & Necessário & Necessário \\
\hline Segurança & Necessário & Necessário \\
\hline Escalabilidade & Desnecessário & Necessário \\
\hline Disponibilidade & Necessário & Necessário \\
\hline Documentação & Riqueza de detalhes menor & Riqueza de detalhes maior \\
\hline
\end{tabular}

\section{Proposta de Ferramenta}

Como foi possível verificar, há um esforço na comunidade científica em construir juízes online para auxiliar o ensino. Porém, ainda há algumas lacunas que precisam ser resolvidas.

Esta seção apresenta a proposta de um juiz online para o ensino de CS1, enfatizando três aspectos (geração de listas, plágio e feedback), que através das experiências, foram considerados prioritários. A Figura 1 apresenta uma visualização da interação do sistema proposto com usuário.

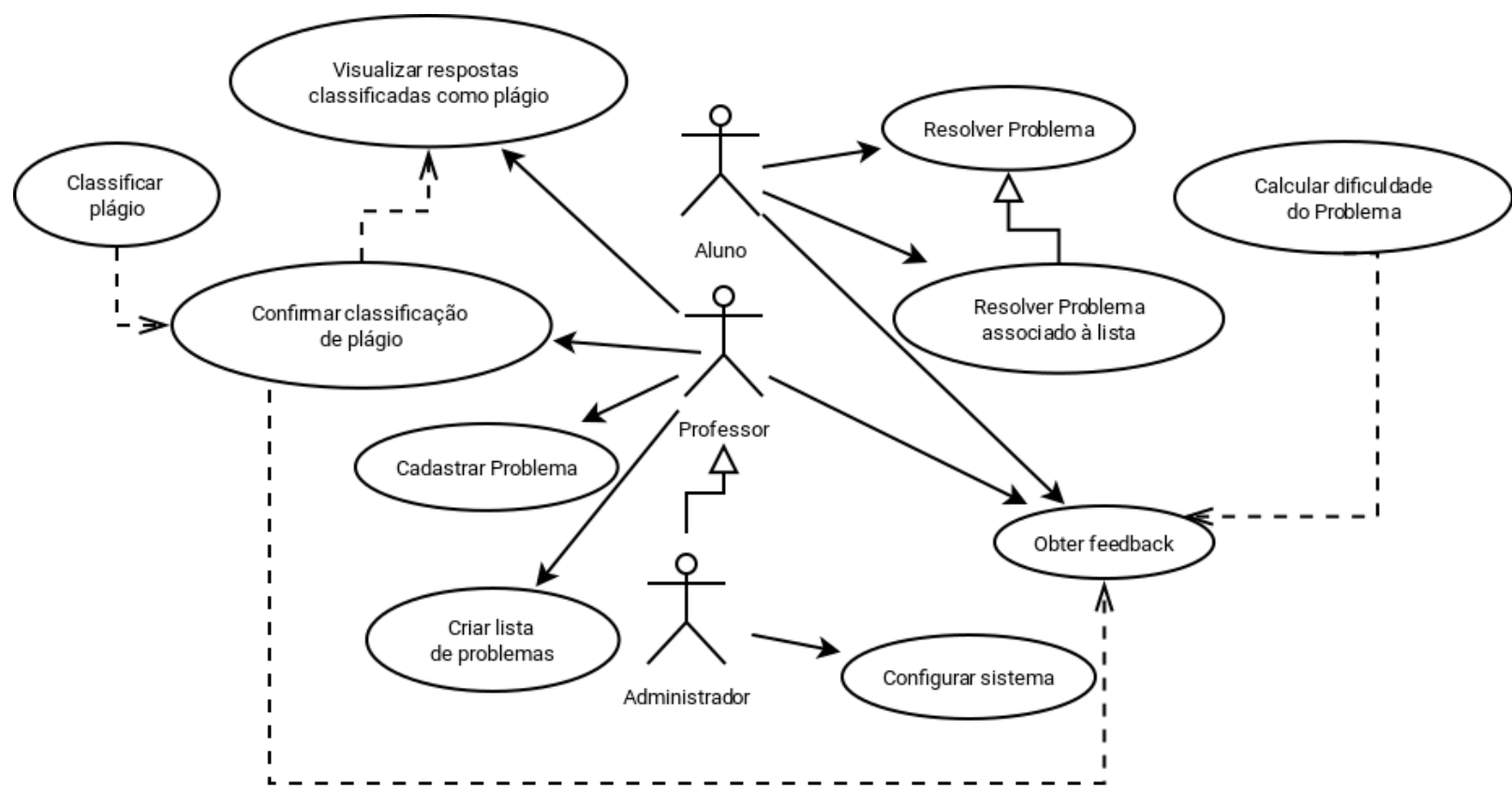

Figura 1: Diagrama de casos de uso para atender aos requisitos

A geração de lista de problemas, de acordo com os parâmetros estabelecidos pelo tutor, só será possível caso, junto ao cadastro do problema, tenha informações extras, tais como:

- O conteúdo abordado (estrutura de repetição, estrutura de seleção, etc.). Desta forma permite que o tutor crie a lista somente com os assuntos já vistos pelos estudantes; 
- O nível de dificuldade do exercício (fácil, médio e difícil). Assim, ao gerar a lista também é possível balancear a dificuldade e fazer um nivelamento da turma;

- Os casos de teste (entrada e saída) como já existem nos juízes online. Neste caso é um cadastro básico para que haja a correção automática e imprescindível em qualquer juiz online.

Atualmente o projeto possui um repositório com 100 exercícios classificados (Francisco \& Ambrósio, 2016). A classificação também levou em consideração atributos como número de linhas de código, número de estruturas de repetição, número de estruturas de seleção, tópicos relacionados, e propriedades sobre a representação dos programas com estruturas de dados. Será possível realizar esta classificação nos próximos exercícios cadastrados considerando as resoluções apresentadas. O aluno poderá resolver exercícios isolados ou relacionados às listas criadas pelos professores. As listas geradas devem ter data de entrega definida e o sistema deve controlar o prazo. Caso o aluno resolva exercícios das listas após o prazo definido, o sistema não associará a pontuação à lista.

Para permitir adaptar a lista ao aluno, deve-se criar um sistema de classificação das habilidades e capacidades do aluno a fim de poder se sugerir exercícios adequados para cada um. Questões de equivalência entre listas geradas para distintos alunos também devem ser levadas em consideração.

O segundo aspecto indispensável para o juiz online aplicado ao ensino de CS1 é a detecção de plágio. Este requisito pôde ser visto como importante tanto na RSL quanto nas experiências com turmas de CS1. A detecção de plágio deve ter as seguintes características:

- Funcionar localmente, de forma que seja integrado ao sistema de correção automática e que seja administrado pela própria ferramenta;

- Classificação semi-automatizada. O algoritmo deve encontrar a possibilidade de plágio e esta deverá ser confirmada pelo tutor que acompanhará as turmas através do juiz online;

- Gerar relatórios com os dados dos estudantes sobre plágio. Assim será possível verificar a relação de plágio individual e também por turmas;

Conforme já apresentado no artigo (Francisco \& Ambrósio, 2016), a classificação do plágio semi-automatizada pode ser realizada através do algoritmo Distância de Edição e técnicas de pré-processamento. Experimentos realizados apontaram que analisar o plágio relacionando turmas e listas de exercícios, trabalhando com probabilidades, pode ser promissor.

O terceiro aspecto é o feedback personalizado. Atualmente os sistemas de correção automática, em geral, possuem um feedback instantâneo que é vantajoso para os estudantes, pois respondem em poucos segundos se o exercício submetido está certo ou errado e, caso esteja errado, ainda é capaz de fornecer outros detalhes (como erro de compilação, resposta errada e limite de tempo excedido). Trata-se de um processo que ocorre em segundos por existir um compilador e um conjunto de testes para cada exercício. Neste caso, espera-se que o juiz online consiga também:

- Realizar uma análise do estilo de codificação do estudante, permitindo um feedback personalizado para este individuo, evitando assim frustração no processo de aprendizagem por demonstrar o erro de forma mais individual;

- Apontar, para um código-fonte com erro de compilação, além do tipo de erro, a linha que pode estar ocasionando este problema. Este tipo de feedback se torna mais possível quando o erro é de compilação;

- Apontar casos de teste que ocasionaram o erro; 
- Treinar aspectos relacionados à metacognição, conforme apresentados por Kautzmann \& Jaques (2016). Desta forma, o estudante terá possibilidade de verificar e aprender com os próprios erros.

Além disso, como feedback aos tutores, pode ser disponibilizado um ranking baseado nas submissões dos estudantes. Este deve levar em consideração o número de submissões e o número de acertos para posicioná-los.

$\mathrm{O}$ administrador do sistema, deve ter acesso às configurações. Será possível configurar a estratégia (algoritmo, normalização, programa externo) para classificação de plágio e definir o conjunto de submissões a serem comparadas (todas as submissões, submissões da turma). Será necessário configurar como calcular a dificuldade dos exercícios (manual, automatizado) e a visibilidade do ranking.

A Figura 2 apresenta um diagrama de classes, que contribui para a construção do software. Diferente do sistema BOCA, que tem entidades bastante relacionadas com competições, o sistema proposto organizará as informações relacionando-as com cursos, disciplinas, turmas e alunos.

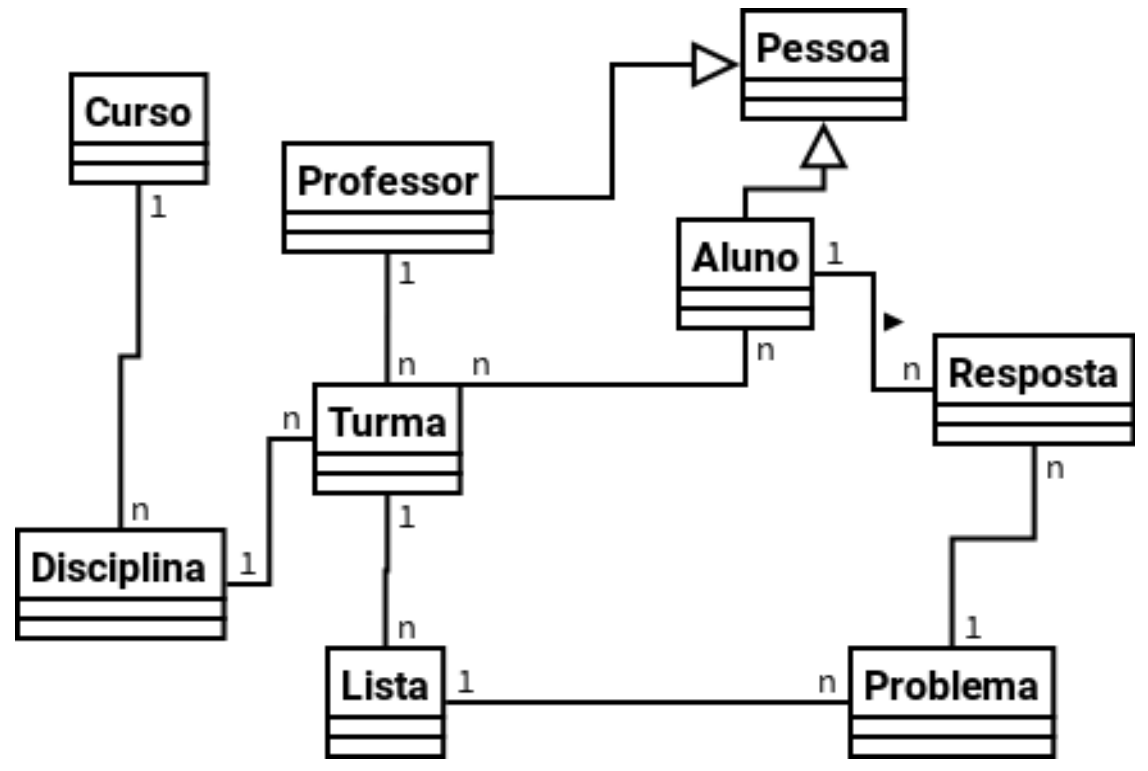

Figura 2: Diagrama de classes para atender aos requisitos

Sabe-se que os requisitos de sistemas surgem conforme uso, o que motiva a criar uma versão inicial do sistema, de acordo com o apresentado, e realizar modificações conforme necessidades de pesquisa, sempre atualizando sua especificação.

A Figura 3 apresenta uma arquitetura preliminar proposta, cujo design considerou requisitos funcionais e não funcionais elencados a partir da RSL e experiência.

A arquitetura foi pensada visando oferecer as funcionalidades descritas aos usuários, alunos e docentes, e possibilitar a integração a outros sistemas, como AVA. Existe um Servidor de Dados que permitirá comunicação com os Servidores de Aplicação (SA). O SA responsável pelo juiz online executará as listas de exercícios, a geração de feedback individual, o cálculo da dificuldade de problemas e a geração de relatórios. O processo de compilar e executar o programa submetido pelo aluno será realizado no Gerador de Feedback Individual. 


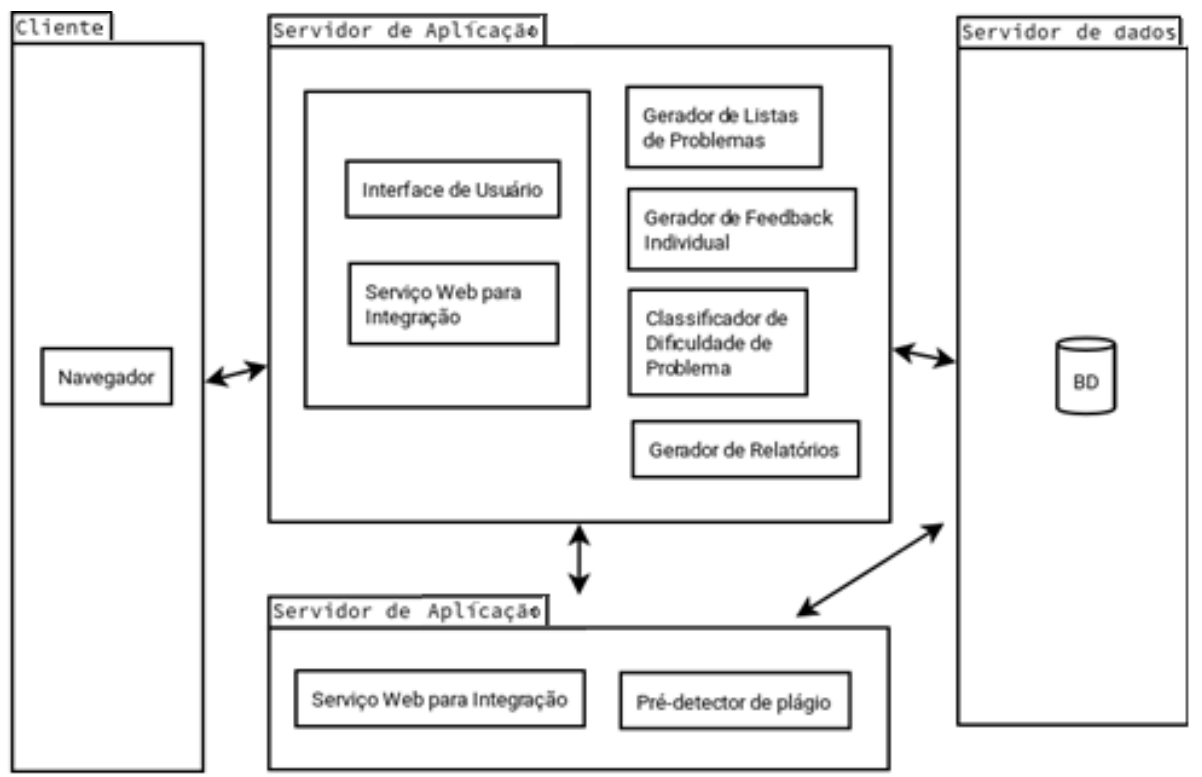

Figura 3: Arquitetura proposta para o sistema

Houve a separação do processamento de plágio, pré-detector de plágio, em outro SA devido a sua alta carga de processamento, e será disponibilizado um Serviço Web para integração com o juiz online. Ao implementar o sistema na arquitetura apresentada, será priorizado atender aos requisitos não funcionais manutenibilidade, segurança, escalabilidade e disponibilidade.

Tabela 3: Comparação do sistema proposto com os demais quanto aos requisitos funcionais

\begin{tabular}{|c|c|c|c|c|}
\hline SISTEMA & Feedback & $\begin{array}{c}\text { Integração com os } \\
\text { Cursos }\end{array}$ & $\begin{array}{c}\text { Desempenho dos } \\
\text { Alunos }\end{array}$ & $\begin{array}{c}\text { Diferentes } \\
\text { Atividades }\end{array}$ \\
\hline Mooshak (E12) & $\mathrm{X}$ & & & \\
\hline AutoLEP (E10) & $\mathrm{X}$ & $\mathrm{X}$ & $\mathrm{X}$ & \\
\hline Egrader (E11) & $\mathrm{X}$ & & $\mathrm{X}$ & \\
\hline FLOP (E8) & $\mathrm{X}$ & $\mathrm{X}$ & & $\mathrm{X}$ \\
\hline Jutge.org (E9) & $\mathrm{X}$ & $\mathrm{X}$ & & $\mathrm{X}$ \\
\hline YOJ (E6) & $\mathrm{X}$ & $\mathrm{X}$ & & $\mathrm{X}$ \\
\hline Athene (E5) & $\mathrm{X}$ & & $\mathrm{X}$ & $\mathrm{X}$ \\
\hline *Experiência UL-FCIS (E3) & $\mathrm{X}$ & $\mathrm{X}$ & $\mathrm{X}$ & $\mathrm{X}$ \\
\hline ViLLE (E1) & $\mathrm{X}$ & $\mathrm{X}$ & $\mathrm{X}$ & \\
\hline ProgTest (E4) & $\mathrm{X}$ & $\mathrm{X}$ & $\mathrm{X}$ & \\
\hline Test MyCode (E7) & $\mathrm{X}$ & $\mathrm{X}$ & & \\
\hline SISTEMA_PROPOSTO & $\mathrm{X}$ & $\mathrm{X}$ & & \\
\hline
\end{tabular}

A Tabela 3 apresenta uma comparação do sistema proposto com os demais sistemas identificados na RSL, considerando os conjuntos de requisitos funcionais. Optou-se por não abordar apenas "Diferentes Atividades", pois há a pretensão de desenvolver as funcionalidades básicas necessárias para o ensino de CS1 e em versões futuras as demais podem ser incluídas, abordando o desenvolvimento da ferramenta de maneira iterativa e incremental.

A Tabela 4 apresenta uma comparação do sistema proposto com os demais considerando os requisitos não-funcionais. A decisão por incluir um detector de plágio trouxe a necessidade de integração, segurança, escalabilidade e disponibilidade. A usabilidade é justificada pela facilidade necessária em sistemas para o ensino. Observa-se que a experiência somada com resultados apresentados pela literatura pode trazer novos requisitos a um juiz online para o ensino de CS1. 
Tabela 4: Comparação do sistema proposto com os demais quanto aos requisitos não-funcionais

\begin{tabular}{|c|c|c|c|c|c|}
\hline SISTEMA & Integ. & Usabil. & Segur. & Escalab. & Disponib. \\
\hline Mooshak (E12) & $\mathrm{X}$ & & & & \\
\hline AutoLEP (E10) & & & & & \\
\hline Egrader (E11) & & & & & \\
\hline FLOP (E8) & & $\mathrm{X}$ & $\mathrm{X}$ & & $\mathrm{X}$ \\
\hline Jutge.org (E9) & & & $\mathrm{X}$ & $\mathrm{X}$ & \\
\hline YOJ (E6) & & & & & $\mathrm{X}$ \\
\hline Athene (E5) & & & & & \\
\hline *Experiência UL-FCIS (E3) & & & $\mathrm{X}$ & $\mathrm{X}$ & \\
\hline ViLLE (E1) & & & $\mathrm{X}$ & & \\
\hline ProgTest (E4) & & & & & \\
\hline Test MyCode (E7) & $\mathrm{X}$ & & & & $\mathrm{X}$ \\
\hline SISTEMA_PROPOSTO & $\mathrm{X}$ & $\mathrm{X}$ & $\mathrm{X}$ & $\mathrm{X}$ & $\mathrm{X}$ \\
\hline
\end{tabular}

\section{Considerações Finais}

O desafio de formar profissionais qualificados em TI relaciona-se com o desenvolvimento das habilidades adquiridas em programação de computadores. A eficácia do trabalho do professor nem sempre é conseguida, dado o tempo necessário para corrigir listas de exercícios e número crescente de alunos. No caso da disciplina de CS1, o uso de sistemas de juiz online tem mostrado que pode contribuir.

No entanto, grande parte dos sistemas de correção automática foram desenvolvidos para competições, e/ou estão disponíveis somente em sites dedicados, o que limita seu uso em sala de aula. Com uma abordagem exploratória, partindo de uma extensa RSL sobre o uso de juiz online no ensino de CS1 e experiências vividas em sala de aula usando o sistema BOCA, o presente artigo teve como objetivo apresentar os requisitos desejáveis em um sistema voltado para o ensino de CS1. O presente projeto de pesquisa teve um viés mais técnico e por isso optou por não estudar detalhadamente a relação entre questões pedagógicas e o uso da ferramenta, no entanto houve um interesse em excluir o aspecto competitividade.

A RSL relacionou benefícios, problemas, requisitos funcionais e requisitos não funcionais em uma perspectiva mais ampla, e a experiência com o sistema BOCA mostrou as necessidades específicas da realidade estudada, trazendo a aprendizagem de algumas lições. Observou-se, por exemplo, as diferenças encontradas nas necessidades de um juiz online para o ensino comparadas com aquelas para competições.

Com a RSL foi possível verificar o problema da falta de validação das ferramentas, o que por muitas vezes dificulta uma comparação dos trabalhos e uma verificação da efetividade de tal metodologia no ensino. Alguns problemas encontrados na RSL também foram vivenciados nas experiências com turmas de CS1. Os requisitos funcionais e não funcionais foram essenciais para propor uma arquitetura de juiz online para o ensino.

A questão da eficácia educacional das ferramentas de juiz online ainda é um problema em aberto. Ramos et al, (2015) mostraram que somente 10,2\% dos artigos avaliados em seu trabalho apresentaram rigor nos resultados, trazendo a validação das ferramentas como um desafio. No entanto, esta RSL apontou diversos benefícios e problemas que podem ocorrer no uso dessas ferramentas. Essa análise é importante para saber quais são os problemas que precisam ser resolvidos e apoiar na busca dos benefícios. Aprendizagem no ritmo do aluno, autoaprendizagem e redução da carga de trabalho do professor são alguns dos benefícios apontados que contribuem não só em ambientes tradicionais de ensino, mas em ambientes de Educação a Distância (EAD) e em MOOC's. A liberdade de definir listas de exercício e a disponibilidade de instrumentos para acompanhar os alunos são questões importantes para o professor. 
A experiência mostrou que há diversos problemas no ensino que passam despercebidos por certos professores, como a alta ocorrência de plágio, e que sistemas para o ensino, por exemplo os juízes online, podem ajudar os docentes a visualizarem estas informações. No entanto, o fato de que não é possível padronizar um software ou linguagem de programação para serem usados em uma disciplina de um modo geral, por todos os professores que a ministram, aumenta a complexidade da tarefa. Esta alta ocorrência de plágio é um sério problema ético e sabe-se que resolvê-lo é bastante desafiador, portanto uma ferramenta que traz tais informações colabora para que o professor busque saber, de alguma forma, o real motivo do plágio, que pode estar ligado, por exemplo, a uma alta sobrecarga de trabalho submetida aos alunos em tempo inviável.

Também foi possível observar pela experiência, a necessidade de geração automática de listas de atividades, sendo este um requisito encontrado também na RSL, o que poderia diminuir ainda mais a carga de trabalho do professor, visto que naturalmente os juízes online já diminuem uma parcela do trabalho ao fazer correções automáticas. Desta forma, a cada assunto abordado seria possível gerar uma lista de exercícios que envolvessem aquele tópico de forma automática.

Confrontar os resultados das duas abordagens enriqueceu a proposta deste trabalho, o que foi de grande valia para definir os requisitos para o sistema proposto e uma especificação preliminar de arquitetura. As experiências com um juiz online desenvolvido prioritariamente para competições trouxeram um complemento necessário para definir quais carências podem apresentar ao usar tal ferramenta no ensino de CS1.

O sistema inicialmente proposto tem o escopo focado em: geração de listas de exercícios, classificação semi-automatizada de plágio e feedback personalizado. Sabe-se da importância de uma arquitetura estável, devido às mudanças de requisitos, para se ter manutenibilidade ao longo do tempo e continuar atendendo aos objetivos do ensino, o que motivou a criar uma arquitetura preliminar que documentou as decisões tomadas.

Com relação ao processo de criação da ferramenta, a primeira estratégia se compôs nas modificações do sistema BOCA, visando tirar o perfil de competição e adaptar aos requisitos necessários para um juiz online voltado ao ensino. Neste aspecto algumas etapas tiveram sucesso, como o feedback do estudante com relação ao nível do exercício (Fácil, médio ou difícil), criação de banco de questões por nível e acesso dos professores à ferramenta. Porém ao tentar implementar, por exemplo, a geração automática de listas de exercícios confrontou-se com o fato de as tabelas e todo o sistema ser do domínio de competições, o que mostrou que uma mudança estrutural nas tabelas e em partes do código-fonte visando contextualizar em cursos e listas de exercícios impactaria em todo o sistema. Isso pôde ser verificado pelo problema enfrentado com o processo de correção automática (autojudge) que passou a não corrigir algumas submissões após o sistema ter sido modificado, onde foi feita a adaptação da estrutura de competições para lidar com as listas de exercícios. Assim, com as experiências levantadas, foi possível observar a necessidade da construção de um novo sistema.

No estágio atual, para o desenvolvimento, vê-se a necessidade de comunidades de pesquisadores e desenvolvedores utilizando uma cultura open-source e colaborativa. Além disso, a colaboração é essencial, neste tipo de projeto, para obter um maior banco de questões e um número grande de usuários, o que permite uma geração de dados suficientes para análise.

Conforme verificado na literatura e já apresentado, há a possibilidade de levantar a qualidade dos programas submetidos pelos alunos, por exemplo com métricas já utilizadas pelos Engenheiros de Software, podendo se relacionar à aspectos como a modularidade, legibilidade e eficiência dos programas. Há também a possibilidade de propor um mecanismo personalizado de adaptatividade do nível de dificuldade dos exercícios, o que traz a oportunidade de explorar, por exemplo, técnicas da Inteligência Artificial. Como essas informações são oriundas de dados subjetivos e contextuais, a pesquisa se torna ainda mais desafiadora, pois é preciso um rigor 
estatístico para aceitar as medidas levantadas. Estes aspectos são propostos como desafios para a comunidade.

A construção e validação deste sistema traz desafios para diferentes áreas do conhecimento (relacionadas à Educação, Estatística e Computação). A integração desses conhecimentos se faz necessária para que se obtenha um produto capaz de chegar às salas de aula e cumprir com a meta de melhorar o ensino de programação.

\section{Agradecimentos}

O presente projeto só foi possível de ser realizado graças ao apoio dado pelo Departamento de TI da Universidade Federal de Goiás. Gostaríamos de agradecer a todos os profissionais envolvidos pelo suporte oferecido.

\section{Referências}

AlShamsi, F., \& Elnagar, A. (2011, April). An automated assessment and reporting tool for introductory Java programs. In Innovations in Information Technology (IIT), 2011 International Conference on (pp. 324-329). IEEE. doi: 10.1109/INNOVATIONS.2011.5893842 [GS Search]

Byrne, P., \& Lyons, G. (2001, June). The effect of student attributes on success in programming. In ACM SIGCSE Bulletin (Vol. 33, No. 3, pp. 49-52). ACM. doi: $\underline{10.1145 / 507758.377467}$ [GS Search]

Bosse, Y., \& Gerosa, M. A. (2015). Reprovações e Trancamentos nas Disciplinas de Introdução à Programação da Universidade de São Paulo: Um Estudo Preliminar. In XXIII WEIWorkshop sobre Educação em Informática. Recife, julho. Disponível em https://drive.google.com/file/d/0BznCaSIV6ocyanVOMlhpNFh6OUk/view?usp=sharing

Campos, C. P., \& Ferreira, C. E. (2004). BOCA: um sistema de apoio a competições de programação. In Workshop de Educação em Computação (pp. 885-895). Disponível em: https://www.ime.usp.br/ cassio/boca/campos-ferreira-wei2004.pdf

Francisco, R. E., \& Ambrósio, A. P. L. (2016). Uso do Algoritmo Distância de Edição com Técnicas de Pré-Processamento para Apoiar a Identificação de Plágio em Códigos-Fonte de Problemas de Programação Introdutória. iSys-Revista Brasileira de Sistemas de Informação, 9(2). Disponível em: http://www.seer.unirio.br/index.php/isys/article/viewFile/5417/5402 [GS Search]

Francisco, R., Júnior, C. P., \& Ambrósio, A. P. (2016). Juiz Online no ensino de Programação Introdutória-Uma Revisao Sistemática da Literatura. In Brazilian Symposium on Computers in Education (Simpósio Brasileiro de Informática na Educação-SBIE) (Vol. 27, No. 1, p. 11). doi: $10.5753 /$ cbie.sbie.2016.11 [GS Search]

Georgouli, K., \& Guerreiro, P. (2010, December). Incorporating an Automatic Judge into Blended Learning Programming Activities. In International Conference on Web-Based Learning (pp. 81-90). Springer Berlin Heidelberg. doi: 10.1007/978-3-642-17407-0 99 [GS Search]

Gomes, A., Areias, C., Henriques, J., \& Mendes, A. J. (2008). Aprendizagem de programação de computadores: dificuldades e ferramentas de suporte. Revista Portuguesa de Pedagogia, (422), 161-179. Disponível em: journals.uc.pt/rppedagogia/article/view/1242/690 [GS Search] 
Kurnia, A., Lim, A., \& Cheang, B. (2001). Online judge. Computers \& Education, 36(4), 299315. doi: 10.1016/S0360-1315(01)00018-5 [GS Search]

Llana, L., Martin-Martin, E., \& Pareja-Flores, C. (2012, November). FLOP, a free laboratory of programming. In Proceedings of the 12th Koli Calling International Conference on Computing Education Research (pp. 93-99). ACM. Disponível em: http://gpd.sip.ucm.es/enrique/publications/koli12/koli12.pdf

Petit, J., Giménez, O., \& Roura, S. (2012, February). Jutge.org: an educational programming judge. In Proceedings of the 43rd ACM technical symposium on Computer Science Education (pp. 445-450). ACM. doi: 10.1145/2157136.2157267 [GS Search]

Pettit, R., Homer, J., Gee, R., Mengel, S., \& Starbuck, A. (2015, February). An Empirical Study of Iterative Improvement in Programming Assignments. In Proceedings of the 46th ACM Technical Symposium on Computer Science Education (pp. 410-415). ACM. doi: $\underline{10.1145 / 2676723.2677279}$ [GS Search]

Pettit, R. S., Homer, J. D., Holcomb, K. M., Simone, N., \& Mengel, S. (2015, June). Are automated assessment tools helpful in programming courses. In 122nd ASEE Annual Conference and Exposition. American Society for Engineering Education (Vol. 2015). doi: $\underline{10.18260 / \text { p.23569 }}$

Poženel, M., Fürst, L., \& Mahnič, V. (2015, May). Introduction of the automated assessment of homework assignments in a university-level programming course. In Information and Communication Technology, Electronics and Microelectronics (MIPRO), 2015 38th International Convention on (pp. 761-766). IEEE. doi: 10.1109/MIPRO.2015.7160373 [GS $\underline{\text { Search] }}$

Rajala, T., Kaila, E., Lindén, R., Kurvinen, E., Lokkila, E., Laakso, M. J., \& Salakoski, T. (2016, February). Automatically assessed electronic exams in programming courses. In Proceedings of the Australasian Computer Science Week Multiconference (p. 11). ACM. doi: $\underline{10.1145 / 2843043.2843062}$ [GS Search]

Ramos, V., Wazlawick, R., Galimberti, M., Freitas, M., \& Mariani, A. C. (2015, October). A Comparação da Realidade Mundial do Ensino de Programação para Iniciantes com a Realidade Nacional: Revisão sistemática da literatura em eventos brasileiros. In Anais do Simpósio Brasileiro de Informática na Educação (Vol. 26, No. 1, p. 318). doi: 10.5753/cbie.sbie.2015.318 [GS Search]

Rocha, B. S., \& França, C. (2016). Obsolescência profissional em engenheiros de software: uma revisão sistemática da literatura. In IX Fórum de Educação em Engenharia de Software. Maringá, Setembro. Disponível em: http://cbsoft.org/cbsoft2016/anais-doseventos/cbsoft2016-fees.pdf

Rountree, N., Rountree, J., Robins, A., \& Hannah, R. (2004, June). Interacting factors that predict success and failure in a CS1 course. In ACM SIGCSE Bulletin (Vol. 36, No. 4, pp. 101-104). ACM. doi: 10.1145/1044550.1041669 [GS Search]

Souza, D. M. D., Isotani, S., \& Barbosa, E. F. (2015). Teaching novice programmers using ProgTest. International Journal of Knowledge and Learning, 10(1), 60-77. doi: $\underline{10.1504 / \text { IJKL.2015.071054 [GS Search] }}$

Sun, H., Li, B., \& Jiao, M. (2014, August). YOJ: An online judge system designed for programming courses. In Computer Science \& Education (ICCSE), 2014 9th International Conference on (pp. 812-816). IEEE. doi: 10.1109/ICCSE.2014.6926575 [GS Search] 
Vier, J., Gluz, J., \& Jaques, P. A. (2015). Empregando Redes Bayesianas para modelar automaticamente o conhecimento dos alunos em Lógica de Programação. Revista Brasileira de Informática na Educação, 23(02), 45.doi: 10.5753/rbie.2015.23.02.45 [GS Search]

Vihavainen, A., Luukkainen, M., \& Pärtel, M. (2013). Test my code: An automatic assessment service for the extreme apprenticeship method. In 2nd International Workshop on Evidencebased Technology Enhanced Learning (pp. 109-116). Springer International Publishing. doi: 10.1007/978-3-319-00554-6_14 [GS Search]

Wang, T., Su, X., Ma, P., Wang, Y., \& Wang, K. (2011). Ability-training-oriented automated assessment in introductory programming course. Computers \& Education, 56(1), 220-226. doi: 10.1016/j.compedu.2010.08.003 [GS Search]

Watson, C., \& Li, F. W. (2014, June). Failure rates in introductory programming revisited. In Proceedings of the 2014 conference on Innovation \& technology in computer science education (pp. 39-44). ACM. doi: 10.1145/2591708.2591749 [GS Search] 